https://helda.helsinki.fi

\title{
Tuning the onset of ferromagnetism in heterogeneous bimetallic nanoparticles by gas phase doping
}

\section{Bohra, Murtaza}

2017-11-09

Bohra , M , Grammatikopoulos , P , Singh , V , Zhao , J , Theodoratou , E , Steinhauer , S , Kioseoglou , J , Bobo , J-F , Nordlund , K, Djurabekova , F \& Sowwan , M 2017 , ' Tuning the onset of ferromagnetism in heterogeneous bimetallic nanoparticles by gas phase doping ' , Physical Review Materials, vol. 1 , no. 6 , 066001 . https://doi.org/10.1103/PhysRevMaterials.1.066001

http://hdl.handle.net/10138/233505

https://doi.org/10.1103/PhysRevMaterials.1.066001

cc_by

publishedVersion

Downloaded from Helda, University of Helsinki institutional repository.

This is an electronic reprint of the original article.

This reprint may differ from the original in pagination and typographic detail.

Please cite the original version. 


\title{
Tuning the onset of ferromagnetism in heterogeneous bimetallic nanoparticles by gas phase doping
}

\author{
Murtaza Bohra, ${ }^{1,2}$ Panagiotis Grammatikopoulos, ${ }^{1, *}$ Vidyadhar Singh, ${ }^{1}$ Junlei Zhao, ${ }^{3}$ Evropi Toulkeridou, ${ }^{1}$ \\ Stephan Steinhauer, ${ }^{1}$ Joseph Kioseoglou, ${ }^{4}$ Jean-François Bobo, ${ }^{5}$ Kai Nordlund, ${ }^{3}$ Flyura Djurabekova, ${ }^{3}$ and Mukhles Sowwan ${ }^{1, *}$ \\ ${ }^{1}$ Nanoparticles by Design Unit, Okinawa Institute of Science and Technology Graduate University, \\ 1919-1 Tancha Onna-Son, Okinawa 904-0495, Japan \\ ${ }_{2}^{2}$ Mahindra Ecole Centrale, Survey Number 62/1A, Bahadurpally Jeedimetla, Hyderabad 500043, Telangana, India \\ ${ }^{3}$ Department of Physics and Helsinki Institute of Physics, University of Helsinki, P. O. Box 43, FIN-00014 Helsinki, Finland \\ ${ }^{4}$ Department of Physics, Aristotle University of Thessaloniki, GR-54124 Thessaloniki, Greece \\ ${ }^{5}$ Centre d'Elaboration de Materiaux et d'Etudes Structurales (CEMES), 29 rue Jeanne Marvig, 31055 Cedex 4 Toulouse, France
}

(Received 10 August 2017; published 9 November 2017)

\begin{abstract}
In the nanoregime, chemical species can reorganize in ways not predicted by their equilibrium bulk behavior. Here, we engineer Ni-Cr nanoalloys at the magnetic end of their compositional range (i.e., 0-15 at. \% Cr), and we investigate the effect of $\mathrm{Cr}$ incorporation on their structural stability and resultant magnetic ordering. To ensure their stoichiometric compositions, the nanoalloys are grown by cluster beam deposition, a method that allows one-step, chemical-free fabrication of bimetallic nanoparticles. While full $\mathrm{Cr}$ segregation toward nanoparticle surfaces is thermodynamically expected for low $\mathrm{Cr}$ concentrations, metastability occurs as the $\mathrm{Cr}$ dopant level increases in the form of residual $\mathrm{Cr}$ in the core region, yielding desirable magnetic properties in a compensatory manner. Using nudged elastic band calculations, residual $\mathrm{Cr}$ in the core is explained based on modifications in the local environment of individual $\mathrm{Cr}$ atoms. The resultant competition between ferromagnetic and antiferromagnetic ordering gives rise to a wide assortment of interesting phenomena, such as a cluster-glass ground state at very low temperatures and an increase in Curie temperature values. We emphasize the importance of obtaining the commonly elusive magnetic nanophase diagram for $M-\mathrm{Cr}(M=\mathrm{Fe}, \mathrm{Co}$, and $\mathrm{Ni}$ ) nanoalloys, and we propose an efficient single-parameter method of tuning the Curie temperature for various technological applications.
\end{abstract}

DOI: 10.1103/PhysRevMaterials.1.066001

\section{INTRODUCTION}

Control of the Curie temperature $T_{C}$, which indicates the paramagnetic-to-ferromagnetic transition, is one of the central concepts in magnetism, and it has been investigated exhaustively over the past half-century $[1,2]$. Yet, following recent advances in nanotechnology, interest in efficient $T_{C}$ control has been rejuvenated in the context of fascinating memory, sensing, and drug delivery properties of magnetic nanoalloys. $M$-Cr nanoalloys (where $M=\mathrm{Fe}, \mathrm{Co}$, and $\mathrm{Ni}$ ), in particular, have been investigated in a large number of experiments on nanoparticles, thin films, core-shell structures, and artificial nanocomposites [3-6]. A major hurdle toward the implementation of such materials, however, is the development of mature technologies for the production of magnetic nanoalloys with well-defined crystalline phase, size, shape, and composition, concurrent with an appropriate magnetic ordering $[7,8]$. The observed deviation of $T_{C}$ values from those of bulk materials has been variously interpreted based on either the oxidation or the segregation paradigm, sometimes even obscuring the difference between the two distinct physical phenomena [7-10].

Among other $M$-Cr alloys, $\mathrm{Cr}$-doped Ni alloys have tunable $T_{C}$ values with minor compositional changes. For instance, slight doping of bulk Ni by $5 \% \mathrm{Cr}$ can set the alloy $T_{C}$ around the human body temperature (315-320 K) [2], which enables direct application in self-controlled heating for hyperthermia

\footnotetext{
*Corresponding authors: pgrammatikopoulos@ oist.jp; mukhles@oist.jp
}

treatment $[4,11]$. However, this unique compositional dependence of $T_{C}$ in the bulk phase may change considerably at the nanoscale due to atomic-scale chemical clustering and/or chemical concentration gradients triggered by various growth and annealing conditions [8-11]; for example, a previous study by the authors suggested high $T_{C}$ values of around $500 \mathrm{~K}$ in $\mathrm{Ni}-5$ at. $\% \mathrm{Cr}$ nanoalloys produced by physical methods, caused by preferential $\mathrm{Cr}$ segregation [8]. On top of this, nanoalloys synthesized following chemical routes require $30 \%$ of $\mathrm{Cr}$ doping (caused by non-negligible size distribution and off-stoichiometry) to achieve bulk Ni-5 at. $\% \mathrm{Cr} T_{C}$ values of $315 \mathrm{~K}$ [11]. Several methods have been suggested to overcome this problem, either by fabricating protected core-shell structures or by fully embedding nanoparticles in a nonmagnetic matrix; such methods have both merits (e.g., protection of surface oxidation) and demerits (e.g., high cost, bio-incompatibility, etc.) $[5,12,13]$. Understanding the growth mechanisms of Ni-Cr nanoalloys is thus crucial to obtain the desired size distribution, compositional homogeneity, and crystalline phase.

Another vital problem of technological interest is the $T_{C}$ behavior in magnetically disordered $\mathrm{Ni}-\mathrm{Cr}$ nanoalloys, where the description of the onset of ferromagnetic order is complicated by the random distribution of both short- and long-range magnetic interactions [ferromagnetic (FM) and/or antiferromagnetic (anti-FM)] [14]. The $T_{C}$ of defect-free $\mathrm{Ni}-\mathrm{Cr}$ alloys is classically described using magnetocrystalline anisotropy (depending on the chemical and crystallographic structure), but at the nanoscale the same mechanistic considerations can lead to surprising morphological (surface and shape anisotropy) and compositional (exchange anisotropy, if 
any) variations [15]. Both from experimental and theoretical viewpoints, Ni-rich $\mathrm{Ni}-\mathrm{Cr}$ nanoalloys form a model system for such an investigation, because of their expected distinctive magnetic behavior emanating from itinerant magnetism synchronized with competing interactions and spatially inhomogeneous magnetization. Superparamagnetism at high temperatures and spin-glass-type behavior at very low temperatures are also anticipated to play some role in these nanoalloys $[7,13]$. The study of incorporating anti-FM Cr dopants $\left(T_{n}=313 \mathrm{~K}\right)$ into $\mathrm{FM} \mathrm{Ni}\left(T_{C}=635 \mathrm{~K}\right)$ is of great interest because it can significantly modify the chemical ordering around the $\mathrm{Ni}$ atoms, thereby optimizing their magnetic properties and paving the way for numerous new applications.

The aim of the present study is to show the effect of $\mathrm{Cr}$ doping in the compositional range $0-15$ at. $\% \mathrm{Cr}$ in $\mathrm{Ni}_{1-x} \mathrm{Cr}_{x}$ nanoparticles fabricated using a cluster beam deposition system. Using various atomistic computer simulation methods, we elucidate the gas-phase growth mechanism and crystalline structure of our nanoparticles, which we correlate with their chemical ordering and resultant magnetic properties. Our one-step, chemical-free fabrication method enables us to tune the $T_{C}$ within a wide temperature range to accommodate the specific needs of particular applications. Most importantly, this is possible by simply controlling a single parameter, i.e., the $\mathrm{Cr}$ concentration of the nanoparticles. We show that a mere overcompensation of 5 at. $\% \mathrm{Cr}$ with respect to bulk compositions yields similar $T_{C}$ values, a phenomenon explained by the fact that full $\mathrm{Cr}$ surface segregation is expected up to this concentration, whereas residual $\mathrm{Cr}$ remains in the nanoparticle cores for doping levels $>5$ at. \%. This is more clearly demonstrated through our constructed nanophase diagram related to both structural and magnetic characteristics, which also reveals a cluster glass magnetic behavior of our samples at low temperatures. Numerous studies are available for compositions $>20$ at. \% Cr for nonmagnetic Ni-Cr superalloys, e.g., nichrome [16]. In this paper, however, we present a detailed compositional study in the magnetic phase of Ni-rich $\mathrm{Ni}-\mathrm{Cr}$ nanoalloys.

\section{EXPERIMENTAL AND COMPUTATIONAL METHODS}

\section{A. Deposition}

$\mathrm{Ni}_{1-x} \mathrm{Cr}_{x}(0 \leqslant x \leqslant 0.15)$ nanoparticles were deposited using a modified dc magnetron-sputtering system (Nanogen50 Source, Mantis Deposition Ltd., UK) [17] onto undoped Si dice and 8-nm-thick $\mathrm{Si}_{3} \mathrm{~N}_{4}$ membrane transmission electron microscopy (TEM) grids. A water-cooled aggregation chamber was evacuated down to $10^{-8}$ mbar prior to sputtering. Constant pressures were maintained at $2.5 \times 10^{-1}$ mbar in the aggregation zone and $6.0 \times 10^{-4}$ mbar in the main chamber with a constant argon (Ar) flow rate set at $60 \mathrm{sccm}$ and a helium $(\mathrm{He})$ flow rate at $5 \mathrm{sccm}$. Sputtering targets of $\mathrm{Ni}-0$, $\mathrm{Ni}-5, \mathrm{Ni}-10$, and $\mathrm{Ni}-15$ at. \% Cr nominal alloy compositions were used. The sputtering power was kept constant at $40 \mathrm{~W}$ for all compositions.

\section{B. Characterization}

After substrate landing, nanoparticle-loaded Si (100) substrates were load-lock transferred to an inert-gas $\left(\mathrm{N}_{2}\right)$ glove box and characterized by atomic force microscopy (AFM) to determine sample coverage. The phase purity of each sample was judged by grazing incidence x-ray diffraction (GIXRD, Bruker D8 Discover $\mathrm{XRD}^{2}$ system with $\mathrm{Cu} K \alpha$ x-ray source) at a grazing angle of $0.25^{\circ}$. Ultrathin carbon film and silicon nitride $\left(\mathrm{Si}_{3} \mathrm{~N}_{4}\right)$ membrane TEM grids were used as substrates for TEM and scanning-TEM (STEM) analysis, using a Cscorrected environmental transmission electron microscope (ETEM, FEI Titan G2 ${ }^{\mathrm{TM}} 80-300 \mathrm{kV}$ ) operated at $300 \mathrm{kV}$. The composition of the nanoparticles was analyzed by electronenergy-loss spectroscopy (EELS) elemental maps using a postcolumn energy filter. X-ray photoelectron spectroscopy (XPS) measurements were performed in a separate ultrahigh-vacuum (UHV) chamber with a base pressure of $2 \times 10^{-9} \mathrm{mbar}$, using a Kratos AXIS Ultra DLD Photoelectron spectrometer with an Al $K \alpha$ anode.

Magnetic properties were measured using a Quantum Design physical property measurement system (PPMS), after correcting for the diamagnetic contribution of the Si substrate used for sample deposition. For zero-field-cooled (ZFC) magnetization, the sample was initially cooled to $5 \mathrm{~K}$ at a zero field, and then magnetizations were measured in the presence of a fixed field upon heating. Subsequently, the field-cooled (FC) magnetization was recorded during cooling in the same field. All samples used for the study of the magnetic properties were fully capped prior to the magnetic measurements using a method previously reported by the authors to prevent surface oxidation [18].

\section{Computer simulation}

Atomic Ni-Ni, $\mathrm{Cr}-\mathrm{Cr}$, and $\mathrm{Ni}-\mathrm{Cr}$ interactions were modeled with an embedded-atom method (EAM) potential by Bonny et al. [19]. Interactions between sputter and inert-gas atoms were represented by a universal, purely repulsive potential [20], whereas Ar atoms interacted with each other using the Lennard-Jones potential [21].

For the nanocluster aggregation study, we used the PARCAS parallel classical molecular-dynamics (MD) code [22-24]. In a cubic box with a 40-nm side length and periodic boundary conditions (PBCs), we introduced $\mathrm{Ni}, \mathrm{Cr}$, and $\mathrm{Ar}$ atoms at random but isolated positions. To sample a wide compositional spectrum (exceeding our experimental $\mathrm{Cr}$ concentrations), we created configurations of 1000 sputter atoms, containing 25, 50 , and 75 at. $\%$ Cr. We introduced 50, 500, or $1000 \mathrm{Ar}$ atoms in the simulation box, corresponding to different inert-gas pressures. The initial temperature of both the sputtered species and the inert-gas atoms was set at $3000 \mathrm{~K}$. However, we demanded that every time inert-gas atoms passed through the simulation box boundaries, their temperatures were set at specific values (see below) using the Berendsen thermostat [25]. For the sputtered atoms, only inert-gas temperature control was used. The nanocluster growth process was decomposed into two sequential phases: plasma zone (PZ) and aggregation zone (AZ) runs; the only difference between them was the thermostat value for the Ar atoms, i.e., 1500 and $300 \mathrm{~K}$ for $\mathrm{PZ}$ and AZ, respectively. PZ simulations ran for 10,100 , and $300 \mathrm{~ns}$, corresponding roughly to various residence times in the condensation chamber. AZ simulations were continuation runs, using the final configurations of the PZ runs as starting points; their running time was up to $300 \mathrm{~ns}$. 
For the MD segregation study and short-range order (SRO) analysis of perfectly crystalline nanoparticles, the method presented in Ref. [8] was followed for nanoparticles 3-7 nm in diameter containing 5, 10, and 15 at. \% Cr. For the segregation study of twinned nanoparticles, MD simulations were performed with LAMMPS [26] in the canonical NVT ensemble, utilizing a Nosé-Hoover thermostat $[27,28]$ at $300 \mathrm{~K}$. The Y-shaped, twinned configuration was constructed by spontaneous relaxation at $300 \mathrm{~K}$ of a nanoparticle deliberately initialized in an unfavorable body-centered-cubic (bcc) structure. MD simulations were complemented by Metropolis Monte Carlo (MMC) runs so as to sample the potential energy landscape more efficiently. A Monte Carlo-based swap of atoms following the Metropolis algorithm was implemented after short sequences of $5 \mathrm{MD}$ steps in the canonical ensemble, where the MD temperature was also used in the Metropolis criterion dictating the swap probability. MMC simulations were up to $\sim 4 \times 10^{6}$ steps long. This combined MD\&MMC method is equivalent to a much longer pure MD simulation.

Transition barriers of the first-nearest-neighbor vacancy jumps were calculated using the nudged elastic band (NEB) method with the climbing image (CI) algorithm [29,30]. The bulk system was built up in an fcc Ni lattice containing $10 \times$ $10 \times 10$ unit cells and full PBC. A vacancy site and a jumping $\mathrm{Cr}$ atom were placed next to each other at the center of the box. In each case, a certain number of surrounding $\mathrm{Ni}$ atoms were substituted by $\mathrm{Cr}$ atoms. Before the NEB calculation, the initial and final images were relaxed to the local energy minima. The NEB calculations were performed by relaxing eight intermediate images using the "quickmin" algorithm [31,32] implemented in LAMMPS [26]. The convergence threshold of the atomic force was set to $10^{-3} \mathrm{eV} / \AA$ and the maximum iterations for both regular NEB and CI were set to 1000 steps. The test runs showed that the variance of the barriers converged to $10^{-5} \mathrm{eV}$ after 300 steps at most.

\section{RESULTS AND DISCUSSION}

\section{A. Nanoparticle growth mechanism}

$\mathrm{Ni}_{1-x} \mathrm{Cr}_{x}(0 \leqslant x \leqslant 0.15)$ nanoparticles were prepared using a modified dc magnetron-sputtering system (see Fig. S1 of the Supplemental Material) [46]. To obtain nanoparticles with well-defined compositions, namely $\mathrm{Ni}-0 \% \mathrm{Cr}, \mathrm{Ni}-5 \%$ $\mathrm{Cr}, \mathrm{Ni}-10 \% \mathrm{Cr}$, and $\mathrm{Ni}-15 \% \mathrm{Cr}$, respective nominal alloy composition targets were used. As a consequence, both atom types, $\mathrm{Ni}$ and $\mathrm{Cr}$, coexisted throughout the whole duration of the nanoparticle nucleation and growth process; alternatively, if two separated single-element targets had been used, the initial cluster seeds would have been monometallic, only to coalesce later in the aggregation process [33].

The inert-gas condensation process can be intuitively explained with the use of classical MD simulations. A 2D schematic representation of our 3D simulation box is shown in Fig. 1, where the $z$-coordinate axis is replaced by one displaying potential energy [34]. $\mathrm{Ni}, \mathrm{Ar}$, and $\mathrm{Cr}$ atoms are represented by red, blue, and green 3D curves [instances (a), (b), and (c), respectively] symbolizing their respective potentials; note that the inert-gas ( $\mathrm{Ar}$ ) potential is purely positive, indicating repulsive forces only, whereas $\mathrm{Ni}$ and $\mathrm{Cr}$ potentials are negative

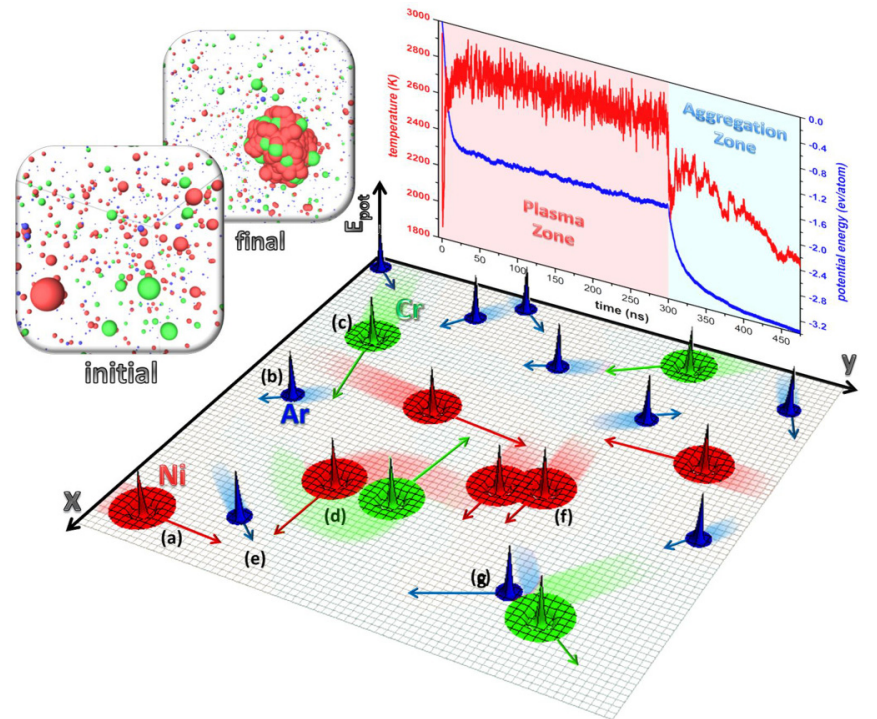

FIG. 1. 2D representation of a 3D simulation box. Ni, Ar, and $\mathrm{Cr}$ atoms are represented by schematics of their potential energies in red [instance (a)], blue [instance (b)], and green [instance (c)], respectively. Arrows indicate velocities of atoms. Instance (g) depicts a $\mathrm{Cr}$ and an $\mathrm{Ar}$ atom postcollision, with the resultant velocity exchange, whereas instance (d) shows temporary mutual attraction of a $\mathrm{Ni}$ and a $\mathrm{Cr}$ atom, which does not result in bond formation. In contrast, instance (e) indicates an imminent three-body collision that may result in bond formation, such as the one shown in instance (f), where a Ni biatom is formed. The temperature of the sputter atoms and their potential energy evolution in a simulation containing $1000 \mathrm{Ar}$, $750 \mathrm{Ni}$, and $250 \mathrm{Cr}$ atoms are shown in the back, explicitly indicating the transition from the plasma zone (PZ) to the aggregation zone (AZ). Initial and final configuration snapshots using the OVITO visualization software [38] are shown on the left, indicating the formation of mixed nascent nanoclusters.

at long distances and positive at short distances; as such, they can produce both attractive and repulsive forces, depending on interatomic distance. The nature of attraction at long distances depends on the specific type of bonding between particular atoms. The strong repulsion at short distances is Pauli-type, caused by the overlap of outer electron orbitals that occurs when atoms get too close to each other [34]. The velocities of the atoms are indicated by arrows of various lengths. Since stable bonds are associated with a decrease in potential energy, only the negative components of the potentials can contribute to nucleation. Ar atoms repel all other atoms; hence, they cannot form any bonds. Instance (g) represents a $\mathrm{Cr}$ and an $\mathrm{Ar}$ atom immediately after collision. Note the exchange of velocities: the $\mathrm{Ar}$ atom is now fast, whereas $\mathrm{Cr}$ has slowed down. $\mathrm{Ni}$ and $\mathrm{Cr}$ atoms are attracted to each other when they feel their attractive potentials. Nevertheless, when two atoms collide, no stable bond can be created; the atoms either crash on each other, eventually bouncing back after feeling the repulsive forces at short distances, or just deviate from their original course [instance (d)]. If, however, their velocities are low enough, a third atom may collide with them and carry away some momentum and energy. When this happens, e.g., in instance (e), the sputtered atoms can be trapped by the attractive component of their respective 
potentials and thus form stable bonds [such as in instance (f)]. This mechanism is known as three-body collision [35-37] and explains why cooling by inert gas is an indispensable mechanism for the nucleation of nanoparticle seeds. Indeed, we ran MD control simulations without any cool inert-gas atoms present, and we found that the temperature of the sputtered atoms always remained too high for any bonding to occur; thus, no nanocluster formation was observed.

The time evolution of the sputter atoms' temperature and average potential energy for an exemplary configuration containing $1000 \mathrm{Ar}, 750 \mathrm{Ni}$, and $250 \mathrm{Cr}$ atoms is also shown in Fig. 1, with a clear distinction between interactions within the hot PZ and the cool AZ. It should be noted that the atomic densities used here do not necessarily agree with experimental ones; this representation is meant to be a qualitative explanation of the physical mechanism of inert-gas condensation (other configurations gave qualitatively similar results with some variation in their numerical values, due to their sensitivity on the intertwined parameters). The initial temperature of all the atoms is $3000 \mathrm{~K}$, but as soon as the Ar atoms pass through the box boundaries, their temperature drops to $1500 \mathrm{~K}$. Consequently, when the (now colder) Ar atoms collide with the $\mathrm{Ni}$ and $\mathrm{Cr}$ atoms, they cool them. Once atoms start bonding to each other, their potential energy decreases (blue curve) and the released energy is transformed into heat, leading to a rise in temperature (red curve). A maximum is eventually reached, depending on the bond formation rate; collisions with Ar atoms not leading to bond formation still cool the system, assisted by collisions that lead to breakups of bonds, counterbalancing the heating. Thus, three contemporary rates determine the temperature of the system: those of bond formation, bond breaking, and elastic collisions. The balance of the effects of these rates can be very delicate. In the simulation presented in Fig. 1, nucleation pauses and the temperature reaches a plateau when newly formed bonds release enough energy to break existing ones. Indeed, in various simulations, we tracked specific atoms and confirmed their rather frequent attaching and detaching to nascent nanoclusters. As a result, after $300 \mathrm{~ns}$ the system is driven out of the PZ and into the AZ before the equilibrium temperature is reached, with an instantaneous drop in temperature due to collisions with room-temperature $\mathrm{Ar}$ atoms. Pronounced clustering resumes, as observed from the upsurge of the temperature curve after the initial temperature drop and, more clearly, from the slope of the potential energy curve. Cooling ensues until the system reaches the equilibrium temperature (i.e., the thermostat temperature of the Ar atoms).

Snapshots indicating the initial and final (after 500 ns) configurations are also depicted in Fig. 1, clearly demonstrating the formation of a random-alloy NiCr nanocluster. In fact, intermediate snapshots (not shown) indicated that mixed element biatoms and nanocluster seeds nucleated from the very beginning. This was true for all configurations studied, and it justifies the use of random solid solutions as starting points for our segregation MD jobs, below. This result, although intuitive, is not self-evident: for example, in a previous study of cosputtering $\mathrm{Ag}$ and $\mathrm{Si}$, no mixed nanoclusters were formed in the primordial growth stage until much later, when preformed single-element nanoclusters coalesced to form Janus nanoparticles [39]. It is also noteworthy that clustering slows down with increasing $\mathrm{Cr}$ concentration, leading to less homogeneous depositions as evident from Fig. S2 of the Supplemental Material [46], which shows snapshots of configurations with various compositions after $300 \mathrm{~ns}$ in the PZ and 300 ns in the AZ. However, it should be stressed that our simulated compositional spectrum is significantly wider than our experimental one, meaning that no such inhomogeneity is expected for our deposited samples; this will be our next point of focus.

\section{B. Crystalline structure and chemical composition analysis}

Knowledge of the size and surface structure of the nanoparticles is significant in order to understand their physical properties. The nanocluster mass distribution dependence on the synthesis parameters was determined in situ by the quadrupole mass filter (QMF) spectrometer, capable of high-resolution measurements and filtering of nanoparticles between $1 \times 10^{3}$ and $4 \times 10^{6}$ amu, prior to the actual depositions; an exemplary comparison of mass variations of selected $\mathrm{Ni}$ and $\mathrm{Ni}-\mathrm{Cr}$ nanoparticle beams is presented in Fig. 2(a). After substrate landing and load-lock transfer, the nanoparticle coverage and size distribution were measured via AFM. Figure 2(b) shows typical AFM topography images and size distributions of $\mathrm{Ni}$ and $\mathrm{Ni}-\mathrm{Cr}$ nanoparticles. We obtained similar height distributions for each of the four depositions, as shown in the insets of Fig. 2(b), showing nanoparticles $5.5 \pm 1.1,5.3 \pm 0.8$, $5.3 \pm 1.0$, and $5.8 \pm 1.4 \mathrm{~nm}$ in diameter for $0 \%, 5 \%, 10 \%$, and $15 \%$ at. $\mathrm{Cr}$, respectively.

Information about the crystalline structure and surface chemical composition of the nanoparticles was obtained by x-ray-based measurements. Figure 2(c) shows GIXRD patterns of $\mathrm{Ni}_{1-x} \mathrm{Cr}_{x}(0 \leqslant x \leqslant 0.15)$ nanoparticles along with International Centre for Diffraction Data (ICDD) records of bulk $\mathrm{Ni}$ and $\mathrm{Ni}-10$ at. \% $\mathrm{Cr}$ alloy for comparison. Three broad peaks located around $2 \theta$ of $44.5^{\circ}, 51.8^{\circ}$, and $76.5^{\circ}$ can be indexed to face-centered-cubic (fcc) Ni planes of [111], [200], and [220], respectively. It is clear that (111) peaks shift toward lower $2 \theta$ angles with increasing $\mathrm{Cr}$ concentration. Lattice constants calculated from the (111) peak width indicate lattice expansion (3.52-3.55 $\AA$ ) compared to the $\mathrm{Ni}$ bulk value $(3.51 \AA)$. Similar lattice expansion was also reported in bulk NiCr alloys [40], but the same was not clearly apparent in reported $\mathrm{NiCr}$ nanoparticles [7] or $\mathrm{NiCr}$ thin films [41] prepared by different growth methods for the given $\mathrm{Ni} / \mathrm{Cr}$ compositions. The increase in lattice sizes can be ascribed to the larger atomic size of $\mathrm{Cr}(1.40 \AA)$ compared to that of $\mathrm{Ni}(1.35 \AA)$, and it indicates the presence of residual $\mathrm{Cr}$ inside the Ni core of the nanoparticles. The average sizes of these nanoalloys, as estimated by the Scherrer equation using (111) peak widths, are in the range of 4-5 nm, comparable with our nanoparticle diameters. This result is counterintuitive considering our previous TEM and MD\&MMC simulation studies [8], which suggested full Cr-surface segregation in 5 at. \% Cr-doped $\mathrm{Ni}$ nanoparticles. However, due to the low $\mathrm{Cr}$ concentrations, such segregation is not clearly discernible by XRD alone. XPS spectra of Ni $2 p$ and $\mathrm{Cr} 2 p$ are shown in Fig. 2(d). XPS quantification in terms of the $\mathrm{Ni} / \mathrm{Cr}$ atomic ratio is consistent with those of parental bulk $\mathrm{Ni}_{1-x} \mathrm{Cr}_{x}(0 \leqslant$ $x \leqslant 0.15)$ compositions. The Ni $2 p_{3 / 2}$ peak, located at binding energy around $857.8-853 \mathrm{eV}$, corresponds to the pure Ni metal, 

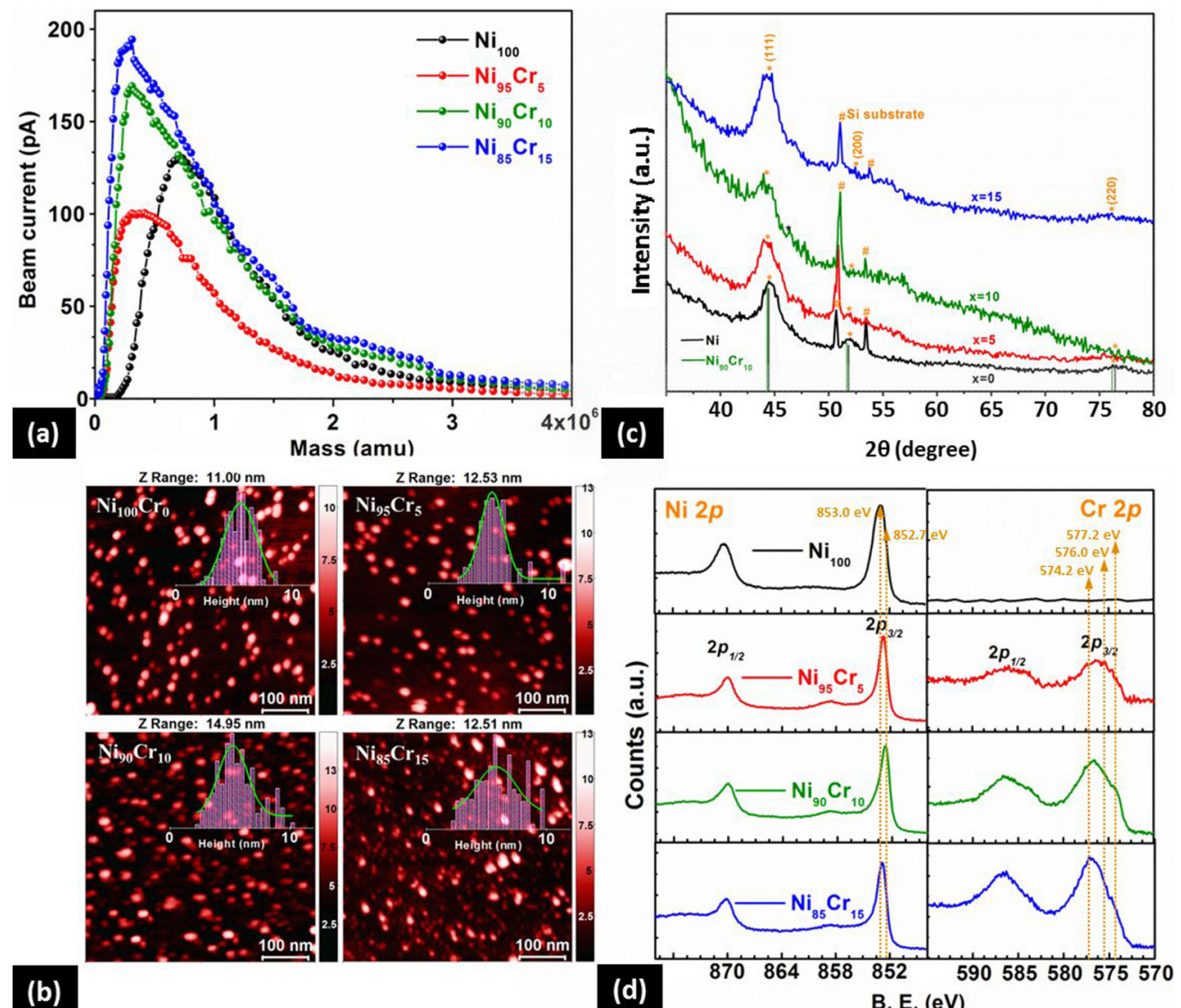

(b)

(d)

B. E. $(\mathrm{eV})$

FIG. 2. (a) QMF profile showing the mass distribution of individual $\mathrm{Ni}_{1-x} \mathrm{Cr}_{x}(0 \leqslant x \leqslant 0.15)$ nanoparticles. (b) AFM topography images and height distributions with fitted Gaussian curves for $\mathrm{Ni}_{1-x} \mathrm{Cr}_{x}(0 \leqslant x \leqslant 0.15)$ nanoparticles. (c) $\mathrm{XRD}$ of $\mathrm{Ni}_{1-x} \mathrm{Cr}_{x}(0 \leqslant x \leqslant 0.15)$ nanoparticles along with ICDD reference data for $\mathrm{Ni}$ and $\mathrm{Ni}-10 \% \mathrm{Cr}$ for comparison. (d) XPS spectra showing the peaks of $\mathrm{Cr} 2 p$ and Ni $2 p$ for $\mathrm{Ni}_{1-x} \mathrm{Cr}_{x}(0 \leqslant x \leqslant 0.15)$ nanoparticles.

while the broad $\mathrm{Cr} 2 p_{3 / 2}$ peak, composed of three energies 577.2, 576, and 574.2 eV, contains contributions by $\mathrm{Cr}^{3+}, \mathrm{Cr}^{2+}$, and metallic $\mathrm{Cr}$, respectively. Since both the deposition and the XPS measurement were performed under high vacuum, surface oxidation was mostly prevented; however, limited $\mathrm{Cr}_{2} \mathrm{O}_{3}$ can also be detected due to air exposure during sample transfer to the XPS chamber.

\section{Cr segregation analysis}

Structural and compositional analysis was also performed at the single nanoparticle level using TEM. Both singlecrystalline and polycrystalline nanoparticles were revealed for all $\mathrm{Cr}$ concentrations studied, including various cases of twinned nanoparticles. Since strain-induced segregation of metallic atoms along grain boundaries (especially incoherent ones) has been reported for bimetallic, multiply twinned nanoparticles [42], emphasis was primarily given to understanding this structure in order to quantify any discernible correlation with the nanoalloy configuration.

Geometric phase analysis (GPA) [43] was implemented to elucidate the structural morphology of an exemplary twinned nanoparticle, shown in Fig. 3(a). The corresponding Fourier transform of Fig. 3(b) is composed of three rotated sets of [110] Ni reflections (spatial frequencies): 200 and 111 spatial frequencies are denoted on the dashed red circle and the interior solid circle, respectively. The three sets of [110] $\mathrm{Ni}$ spatial frequencies correspond to three rotated grains of the $\mathrm{Ni}$ structure in the nanoparticle. The three grains are denoted $a, b$, and $c$ on the experimental image, and the corresponding amplitude image of the 200 spatial frequency of the $a$ grain is given in Fig. 3(c). Amplitude images are equivalent to dark-field images using the corresponding spatial frequency in the Fourier transform; hence, the grain boundaries are directly revealed. It is obvious that the $\mathrm{Ni}$ nanoparticle contains three grains whose boundaries form a Y shape, as shown in Fig. 3(c). Taking into account the crystallographic data of $\mathrm{Ni}$ and $\mathrm{Cr}$ crystals, the diffraction patterns of all the high-symmetry directions were simulated. Peak finding technique [44] measurements of distances and angles between planes on the experimental image as well as on the Fourier transform concluded that the zone axis of the nanoparticle is the [110] of the Ni structure; the corresponding simulated diffraction pattern is shown in Fig. 3(d), performed using the EMS-JEMS software [45]. In Figs. 3(e)-3(g), the 200 phase image of grain $a$ and the 111 phase images of grains $b$ and $c$ are depicted, elucidating the formation of the triple grain boundary. The corresponding spatial frequencies of the three grains are denoted by different colors on the Fourier transform of Fig. 3(b): yellow, blue, and green for grains $a, b$, and $c$, 

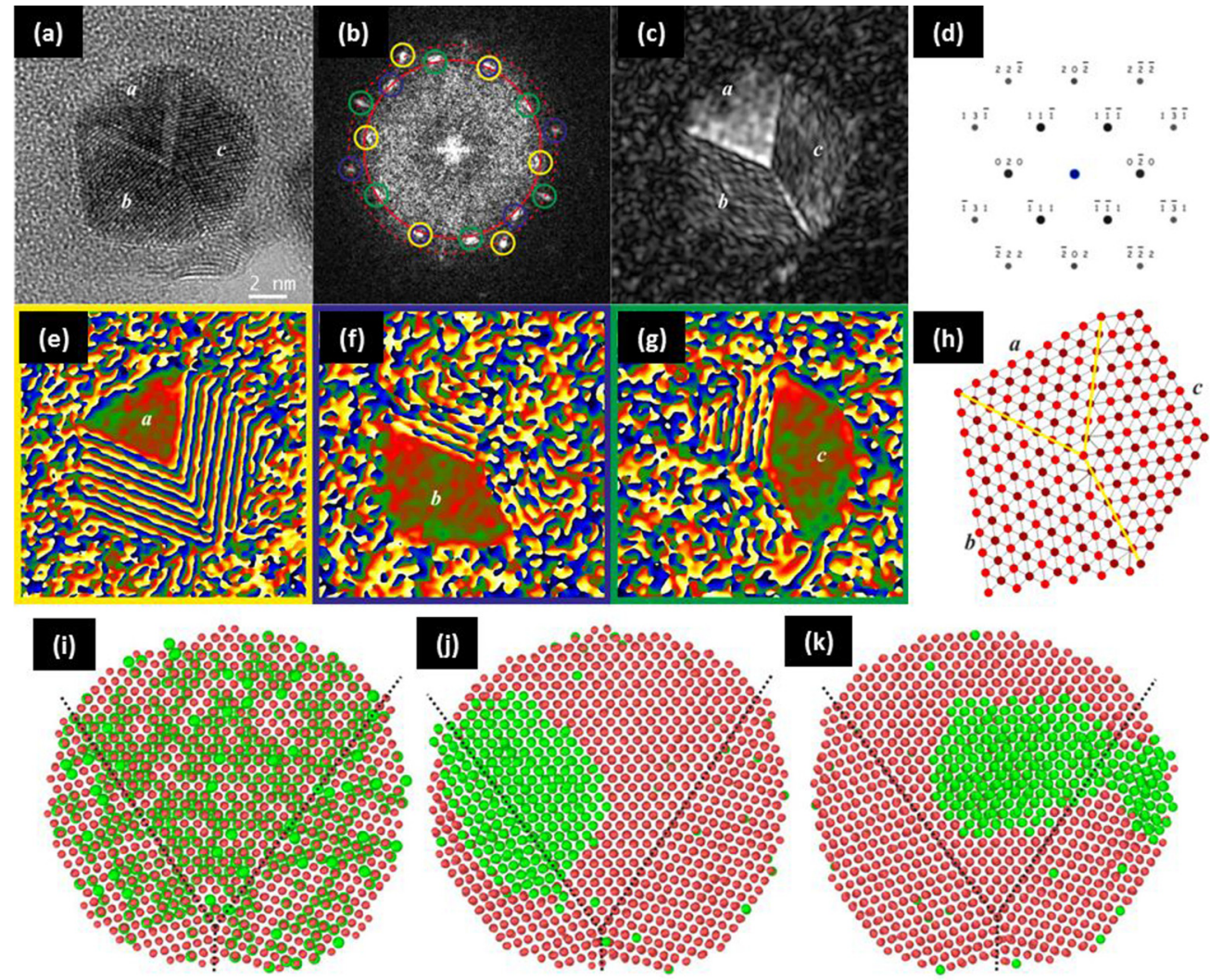

FIG. 3. (a) TEM image of multiply twinned nanoparticle, where three grains, $a, b$, and $c$ are designated. (b) Corresponding Fourier transform, where the corresponding spatial frequencies of the three grains are denoted by different colors: yellow, blue, and green for grains $a, b$, and $c$, respectively. (c) Amplitude image of the 200 spatial frequency of grain $a$, as calculated by the GPA. (d) Corresponding simulated diffraction pattern of the [110] zone axis of the Ni structure. (e) Phase images of the 200 spatial frequency of grain $a$. (f),(g) 111 phase images of grains $b$ and $c$, respectively. (h) Atomistic model of the multiply twinned nanoparticle projected along the [110] direction. The three grain boundaries forming a Y shape are denoted by a yellow line. Different shades of atoms denote different heights along the projection direction. (i) Initial configuration of a Y-shaped twin boundary in a Ni nanoparticle, where 5\% of the atoms were replaced randomly by $\mathrm{Cr}$ atoms. (j)-(k) Two exemplary final configurations of combined MD\&MMC simulations, where the $\mathrm{Cr}$ atoms form satellites on the nanoparticle surfaces. Red spheres represent $\mathrm{Ni}$ atoms, while blue spheres represent $\mathrm{Cr}$ atoms.

respectively. It is noted that grains $a$ and $b$ have a common set of 111 spatial frequencies, and hence they form a coherent twin boundary plane. The other two boundary planes are not coherent; crystallographic analysis concluded on the (111) plane of grain $a$ for the $a c$ twin boundary, and the (111) plane of grain $c$ for the $b c$ twin boundary. Taking into account the aforementioned analysis and results, an atomistic model of the triple-grained nanoparticle was constructed, as presented in Fig. 3(h) along the [110] direction. The three grain boundaries forming the $\mathrm{Y}$ shape are indicated by a yellow line. A coherent twin boundary between grains $a-b$ is revealed, as well as two incoherent boundaries between grains $a-c$ and $c-b$, formed with a series of strained bonds between $\mathrm{Ni}$ atoms.

Simulating surface segregation in twinned nanoparticles allowed us to resolve whether the presence of twins affects the segregation or not. In Fig. 3(i), the initial configuration of a Y-shaped twin boundary is presented. For our configuration to be directly comparable with those presented in Ref. [8], where perfectly monocrystalline nanoparticles alone were examined, $5 \%$ of the atoms of the initial $\mathrm{Ni}$ nanoparticle were replaced randomly by $\mathrm{Cr}$ atoms. Several different sets of randomly distributed $\mathrm{Cr}$ atoms were tested with different initial velocity distributions at $300 \mathrm{~K}$ to elucidate the effect of the twin boundaries on the $\mathrm{Cr}$ segregation. In Figs. 3(j) and $3(\mathrm{k})$, two exemplary final configurations of combined MD\&MMC simulations are shown. It is clear that in all cases, $\mathrm{Ni}$-core-Cr-satellite configurations emerge, analogous to those reported in our previous study of perfectly monocrystalline nanoparticles. The twin boundaries were found to neither pin or obstruct $\mathrm{Cr}$ atoms, nor help their segregation toward the outer surface of the nanoparticle. In all cases, $\mathrm{Cr}$ forms satellites at random positions with respect to the Y-shaped twin boundary. Hence, it can be deduced that the frequently observed low-energy twin boundaries in $\mathrm{NiCr}$ nanoparticles do not affect the formation of the $\mathrm{Cr}$ satellites, presumably due to the small atomic size difference between $\mathrm{Ni}$ and $\mathrm{Cr}$.

Once the effect of the twin boundaries on $\mathrm{Cr}$ segregation was found to be negligible, a combination of MD\&MMC simulations was subsequently employed to investigate the preferential surface segregation (or lack thereof) of $\mathrm{Cr}$ in nanoparticles of higher $\mathrm{Cr}$ concentration than those reported in Ref. [8]. A detailed segregation study of $\mathrm{Cr} 5-20$ at. $\%$ showed that full surface elemental segregation is still expected, as the thermodynamically stable structure is of a 

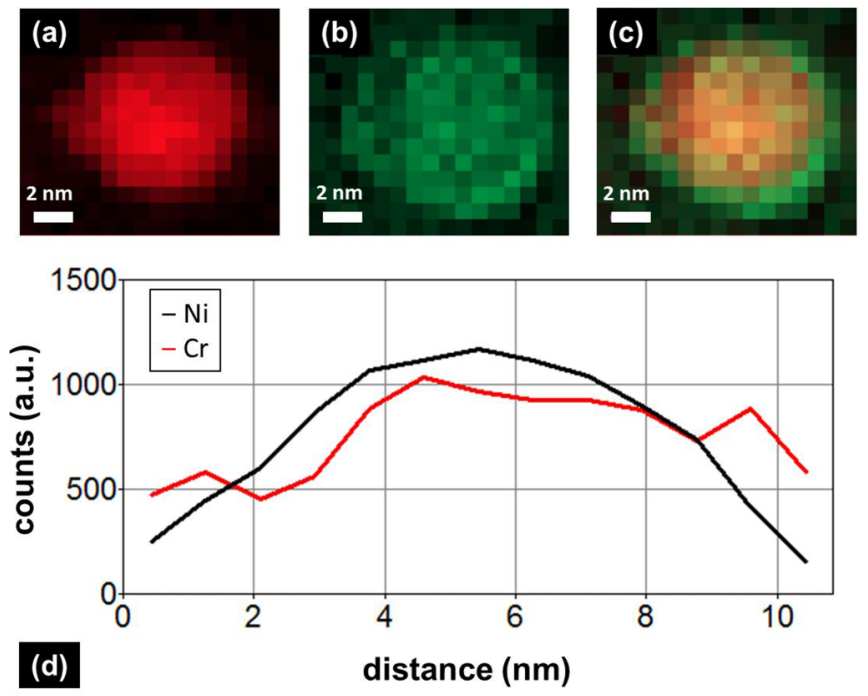

FIG. 4. Elemental mapping of a single $\mathrm{NiCr}$ nanoparticle by EELS (15 at. \% Cr case): (a) EELS signal of $\mathrm{Ni} L_{2,3}$ edge (scale bar $2 \mathrm{~nm}$ ). (b) EELS signal of Cr $L_{2,3}$ edge (scale bar $2 \mathrm{~nm}$ ). (c) Combination of (a) and (b). (d) Line profile from left to right (width four pixels) suggests residual $\mathrm{Cr}$ in the nanocluster core and $\mathrm{Cr}$ enrichment toward the nanocluster surface, rather than a distinct Ni-core/Cr-shell morphology.

segregated Ni-core-Cr-satellite arrangement (see Fig. S3 of the Supplemental Material) [46]. Indeed, for the case of 5 at. $\%$ Cr-doped Ni nanoparticles, polycrystalline particles with Cr-rich partial shells were observed by TEM, as previously reported in Ref. [8]. In contrast, elemental mapping by EELS revealed different $\mathrm{NiCr}$ nanoparticle morphologies for the samples with 10 and 15 at. \% Cr. Representative results for elemental mapping of a single $\mathrm{NiCr}$ nanoparticle (15 at. \% $\mathrm{Cr}$ case) are shown in Figs. 4(a) and 4(b) for the Ni $L_{2,3}$ and $\mathrm{Cr} L_{2,3}$ edges, respectively [Fig. 4(c) is a combination of Figs. 4(a) and 4(b)]. Although the corresponding line profiles [Fig. 4(d)] do show Cr enrichment toward the nanocluster surface, residual $\mathrm{Cr}$ can be expected in the core region since the maximum of its signal appears around the center, which corroborates the XRD results described above.

Therefore, the experimentally obtained structures cannot be explained by energetic arguments alone; kinetics aspects must also be taken into account, especially considering that the employed deposition method has been known to produce kinetically trapped, metastable configurations [33]. Phase segregation due to thermally activated atomic diffusion happens slowly, typically at the microsecond scale, meaning that residual Cr can be trapped in the nanoparticle core as a result of sluggish segregation inside the aggregation zone, or quenching inside the deposition chamber; naturally, this is more likely to happen at nanoparticles of higher $\mathrm{Cr}$ concentration. SRO analysis of MD simulations of $\mathrm{Ni}_{95-85} \mathrm{Cr}_{5-15}$ nanoparticles 3,5 , and $7 \mathrm{~nm}$ in diameter at various temperatures $(600-$ $1200 \mathrm{~K})$ verifies this suggestion, as shown in Fig. S4 of the Supplemental Material [46]. Most interestingly, it can be seen that the precipitation rate decreases by almost $50 \%$ with an increase of $\mathrm{Cr}$ concentration from 5 to 15 at. \%, implying that there is another, more substantial difference between the two

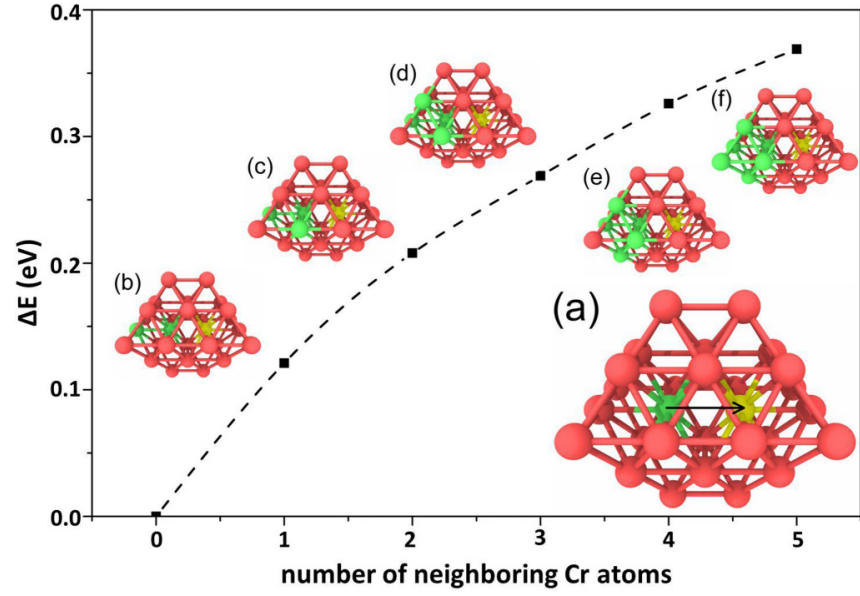

FIG. 5. 1NN vacancy migration considering the atomic local environment, including second-nearest-neighbor ( $2 \mathrm{NN})$ atoms: the $\mathrm{Ni}$ and $\mathrm{Cr}$ atoms and the vacancy site are colored in red, green, and yellow, respectively. The migration path is shown in the enlarged inset (a). The configurations in insets (b)-(f) correspond to increasing numbers of neighboring $\mathrm{Cr}$ atoms (from 1 to 5 ).

structures. Indeed, in the latter, the probability for a $\mathrm{Cr}$ atom to be first-nearest-neighbor (1NN) of another $\mathrm{Cr}$ atom is significantly higher, since the nanoparticles assume overall fcc lattice structures, i.e., they have $121 \mathrm{NNs}$ around each lattice site; this fact can have a profound effect on the segregation process.

To understand the nature of migration of $\mathrm{Cr}$ atoms within the Ni lattice better, we analyzed energy barriers that can determine the kinetics of the segregation processes in $\mathrm{NiCr}$ nanoparticles. NEB calculations show that the energy barrier for a single $\mathrm{Cr}$ atom to jump to the $1 \mathrm{NN}$ vacant site in a pure Ni matrix is $0.680 \mathrm{eV}$. We gradually increased the number of $\mathrm{Cr}$ atoms near the jumping atom to analyze the change in the energy barrier for a $\mathrm{Cr}$ atom to leave a $\mathrm{Cr}$ precipitate with increasing size. By a jump, we always assume that the $\mathrm{Cr}$ atom leaves the $\mathrm{Cr}$ precipitate. This is the process with the highest energy barrier that is well defined and can be compared between the different structures. In Fig. 5 and Table I, we present the barriers for forward $\left(E_{m}^{f}\right)$ and reverse $\left(E_{m}^{r}\right)$ transitions, which are defined as the energy difference between the highest replica and the first and last replicas in the NEB calculations, respectively. The difference between the two barriers,

TABLE I. Typical forward $\left(E_{m}^{f}\right)$ and reverse $\left(E_{m}^{r}\right)$ migration energy barriers (in eV) of $\mathrm{Cr} 1 \mathrm{NN}$ vacancy jump, where one $\mathrm{Cr}$ atom jumps from the $\mathrm{Cr}$ precipitate into the $\mathrm{Ni}$ matrix. Energy differences $(\Delta E)$ between the initial and final replicas indicate that the stability of $\mathrm{Cr}$ precipitates depends on their size.

\begin{tabular}{lccc}
\hline \hline $\begin{array}{l}\text { Number of neighboring } \\
\text { Cr atoms }\end{array}$ & $E_{m}^{f}(\mathrm{eV})$ & $E_{m}^{r}(\mathrm{eV})$ & $\Delta E(\mathrm{eV})$ \\
\hline 0 & 0.680 & 0.680 & 0 \\
1 & 0.752 & 0.631 & 0.121 \\
2 & 0.786 & 0.578 & 0.208 \\
3 & 0.769 & 0.500 & 0.269 \\
4 & 0.820 & 0.494 & 0.326 \\
5 & 0.863 & 0.494 & 0.369 \\
\hline \hline
\end{tabular}


$\Delta E=E_{m}^{f}-E_{m}^{r}$, shown in the last column of Table I, corresponds to the energy difference in the initial and the final states; note that this difference is always positive (i.e., the barrier for a $\mathrm{Cr}$ atom to return back to the precipitate is lower than the one to leave), and that the two $\mathrm{Cr}$ atoms already prefer to stay together with an energy difference $\Delta E=0.121 \mathrm{eV}$. However, this does not necessarily indicate that the precipitate itself is stable. On the contrary, the $\mathrm{Cr}$ atom pair appears to be rather mobile, since the low reverse barrier is the same for both atoms of the pair. The energy difference is further increased with an increase of the $\mathrm{Cr}$ precipitate size. This positive correlation is illustrated in Fig. 5, where specific scenarios of barrier calculations are shown. We correlate the tendency seen in Fig. 5 with the stability and immobility of the small precipitates. We also calculated the energy barriers for $\mathrm{Ni}$ atoms to exchange with the vacancy near the $\mathrm{Cr}$ precipitate so as to separate the emitted atom from the precipitate: these barriers are always found around $0.90-1.25 \mathrm{eV}$. More generalized configurations were also considered (see Fig. S5 of the Supplemental Material [46]). The difference between the mean values of $\mathrm{Cr}$ and $\mathrm{Ni}$ jump barriers is $0.288 \mathrm{eV}$. Hence, it is rather unlikely that the $\mathrm{Ni}$ atom will move between the $\mathrm{Cr}$ atoms to promote the further moving of the $\mathrm{Cr}$ atom away from the precipitate. On the contrary, a $\mathrm{Cr}$ atom that left the precipitate has a higher probability to return to it, while the migration barriers for other $\mathrm{Cr}$ atoms from the precipitate are also rather high. Even though these are not as high as the barrier for a $\mathrm{Ni}$ atom, their values are increasing with larger $\mathrm{Cr}$ precipitate sizes, contributing to the reduced mobility of small $\mathrm{Cr}$ precipitates, which can remain as residue inside the $\mathrm{Ni}$ core of the nanoparticles.

\section{Tuning the Curie temperature via $\mathrm{Cr}$ concentration modification}

The main goal of this work was to obtain a system that preserves the onset of ferromagnetism of bulk $\mathrm{NiCr}$ at the nanoscale for targeted applications; to investigate this, we carried out FC magnetization measurements between $5 \leqslant$ $T \leqslant 700 \mathrm{~K}$ in a constant applied field of $H_{\text {app }}=1 \mathrm{kOe}$, as shown in Fig. 6(a). For comparison, the measured $T_{C}$ 's of bulk target alloys with corresponding compositions are also indicated. Clearly, $T_{C}$ values, indicating where spontaneous magnetization tends to become zero, steadily decrease with increasing $\mathrm{Cr}$ concentration; however, they consistently remain higher than those of the corresponding parent bulk alloys of the same compositions.

The drift of $T_{C}$ values toward lower temperatures with increasing $\mathrm{Cr}$ concentration is more clearly demonstrated in Fig. 6(b), which shows their probability distribution functions as obtained from FC $M(T)$ graphs normalized over the lowest temperature magnetization measurement [i.e., $M(5 \mathrm{~K})$ ]; details can be found in Fig. S6 of the Supplemental Material [46]. The obtained values are 620, 550, 340, and $150 \mathrm{~K}$ for $0,5,10$, and 15 at. $\% \mathrm{Cr}$, respectively. Notably, the standard deviations of the distributions of the alloy samples do not change significantly, indicating that the samples preserve their structure in a similar fashion during the measurement regardless of $\mathrm{Cr}$ concentration. Most importantly, the approximately linear, monotonic character of the drift readily enables fine-tuning the $T_{C}$ within a wide temperature range by simply
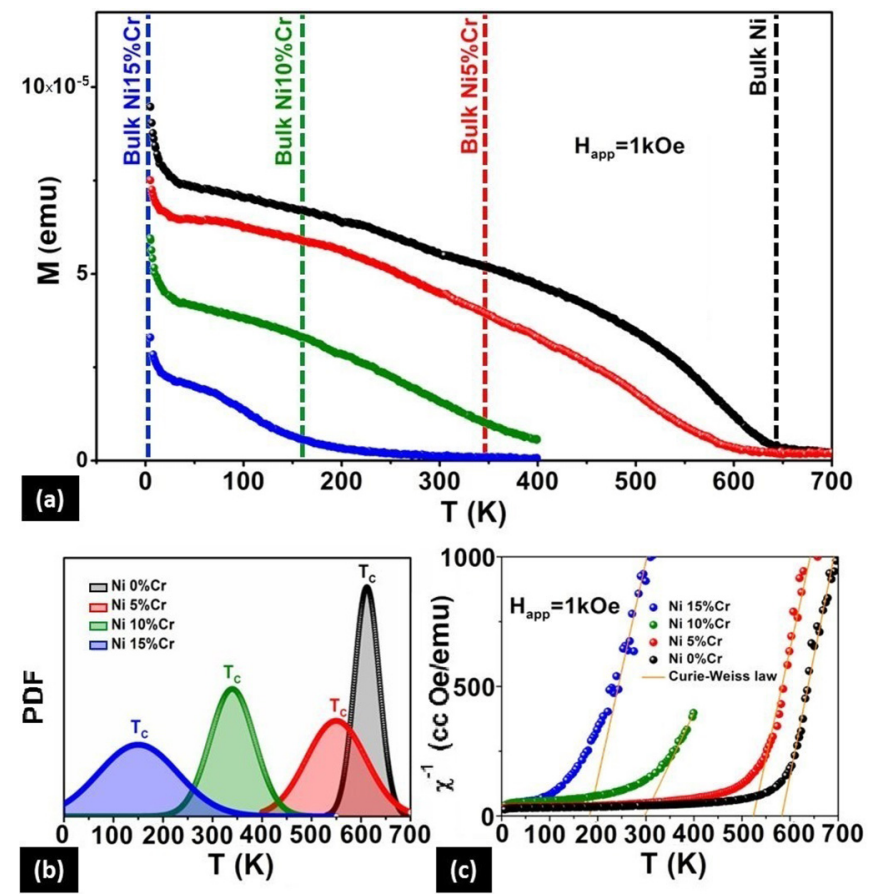

FIG. 6. (a) FC magnetizations vs temperature under a magnetic field of $1 \mathrm{kOe}$ in the temperature range $5 \leqslant T \leqslant 700 \mathrm{~K}$. The $T_{C}$ values of bulk $\mathrm{Ni}_{1-x} \mathrm{Cr}_{x}$ alloys are also indicated for comparison. (b) Curie temperature probability distribution functions obtained from normalized FC magnetization vs temperature graphs under 1 $\mathrm{kOe}$, indicating a clear shift toward lower values with increasing $\mathrm{Cr}$ concentration. (c) Corresponding $1 / \chi$ vs $T$ curves for the $\mathrm{Ni}_{1-x} \mathrm{Cr}_{x}$ $(0 \leqslant x \leqslant 0.15)$ nanoparticles. The red lines represent fitted data to the modified Curie-Weiss law in the paramagnetic region.

modifying a single parameter, that of $\mathrm{Cr}$ concentration; we will return to this key point later.

To examine whether in the pure paramagnetic regime the magnetization follows the Curie-Weiss (CW) law [47], corresponding inverse susceptibility $(1 / \chi)$ versus $T$ curves are plotted in Fig. 6(c) for samples of all compositions under study. We analyze the data using a modified $\mathrm{CW}$ dependence [48],

$$
\frac{1}{\chi}=\frac{T}{C}\left(1-\frac{\theta}{\gamma} \frac{1}{T}+\cdots\right)^{\gamma},
$$

where $C$ is the Curie constant and $\theta$ is the paramagnetic Curie temperature. The truncated form of this expression (up to the $1 / T$ term) yields the Curie point $T_{C}=\frac{\theta}{\gamma}$ (i.e., the temperature at which $1 / \chi$ vanishes). This is different from well-known mean-field results, i.e., $T_{C}=\theta$, by an extra factor of $1 / \gamma$ (here $\gamma=1.396$, which corresponds to the 3D Heisenberg universality). Because of this factor, one can measure the $T_{C}$ with an accuracy of $\pm 15 \%$; otherwise a mean-field approximation may overestimate the $T_{C}$ value, commonly by a factor of 1.66 [48]. We find that in the high-temperature regime, all $\mathrm{Ni}_{1-x} \mathrm{Cr}_{x}(0 \leqslant x \leqslant 0.15)$ nanoparticles obey the CW law; however, some deviation occurs at temperatures just above their respective transition temperature, which can be attributed to $\mathrm{Cr}$ segregation. 
It should be noted that our method entails room-temperature deposition with no need for high processing temperatures that could have a detrimental effect on the homogeneity of our samples. A $T_{C}$ value near the human body temperature can be observed for our $\mathrm{Ni}_{90} \mathrm{Cr}_{10}$ sample; by linear interpolation we deduce that $\mathrm{Ni}_{89} \mathrm{Cr}_{11}$ would have been even closer. Such a result is valuable for self-regulating, magnetic fluid hyperthermia treatment, due to the relatively narrow size distribution of our nanoparticles. Consequently, it can be expected that results of calorimetric measurements would not exceed the therapeutic Curie point of $318 \mathrm{~K}$, unlike previously reported $\mathrm{Ni}-\mathrm{Cr}$ nanoparticles synthesized by a chemical route, displaying large heterogeneity both in size and composition [11]. Beyond hyperthermia treatment, however, $\mathrm{Ni}_{1-x} \mathrm{Cr}_{x}$ nanoparticles with tunable $T_{C}$ can be used as a supporting substrate for Ni-based taps and magnetic resonance imaging applications, where reduced ferromagnetism is desirable to avoid unwanted hysteresis losses [49]. Further, controlling the $T_{C}$ around room temperature may pave the way for more advanced magnetocaloric applications of $M-\mathrm{Cr}$ nanoclusters: for instance, miniaturized versions of thin film devices such as for coolers on chips $[49,50]$.

\section{E. Determination of the magnetic state}

It should be stressed here that the $T_{C}$ is an average macroscopic property; therefore, identifying its value for each sample may be important, but it does not fully describe the structure and various competitive interactions present inside a system consisting of magnetic nanoparticles. To understand the magnetic state of our samples, ZFC and FC magnetization curves under low magnetic field (i.e., $50 \mathrm{Oe}$ ) are shown in Fig. 7(a) within the temperature range 5-400 K. The ZFC and FC magnetizations of each sample show a considerable divergence at an irreversibility temperature, $T_{\mathrm{irr}}$, defined as the point where $M_{\mathrm{FC}}\left(T_{\text {irr }}\right) \neq M_{\mathrm{ZFC}}\left(T_{\text {irr }}\right)$. Irrespective of the sample, ZFC magnetizations increase during cooling to the magnetic transition temperature $\left(T_{S}\right)$, estimated from the maxima in ZFC curves, and then show a downturn below it. The FC magnetizations consistently retrace the ZFC magnetizations above $T_{S}$, but irreversibility in magnetization occurs around $T_{\mathrm{irr}} \geqslant T_{S}$. The $T_{S}$ values decrease as the $\mathrm{Cr}$ concentration increases. Qualitatively, it is evident from the ZFC-FC curves that magnetization decreases with the addition of $\mathrm{Cr}$. This suggests that the volume fraction of FM contributions decreases as $\mathrm{Cr}$ concentration is increased. Differences between ZFC and FC magnetization curves and the maxima $\left(T_{S}\right)$ in $\mathrm{ZFC}$ curves are commonly credited to the existence of canonical spin glass, cluster glass, or superparamagnetic phases [50]. However, FC magnetization below $T_{\text {irr }}$ is not constant here, as in canonical spin glasses, but continues to increase with decreasing temperature. Moreover, the irreversibility in the present case occurs in closer vicinity of $T_{\mathrm{S}}\left(T_{\mathrm{irr}} \approx T_{S}\right)$ with increasing $\mathrm{Cr}$ content, unlike in ideal superparamagnetic systems [51], where it is expected to start well above $T_{\mathrm{S}}\left(T_{\mathrm{irr}}>T_{S}\right)$. This behavior normally indicates cluster glass features of concentrated magnetic systems and magnetic disorder [51,52], or a superparamagnetic material with dipolar interactions [51,52]. Thus, the samples may have complex magnetic phases with coexisting anti-FM-FM
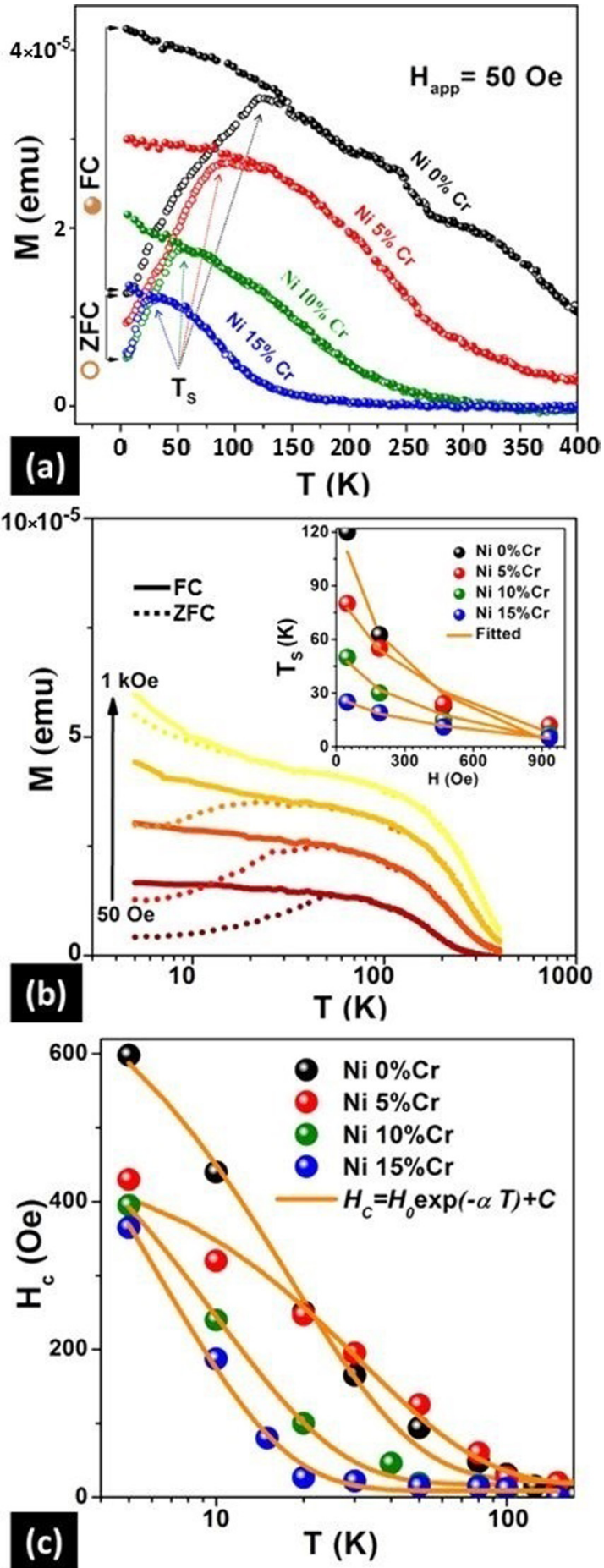

FIG. 7. (a) ZFC and FC magnetization curves for the $\mathrm{Ni}_{1-x} \mathrm{Cr}_{x}$ $(0 \leqslant x \leqslant 0.15)$ nanoparticles under a low magnetic field of $50 \mathrm{Oe}$. (b) Field-dependent ZFC and FC magnetization measured for a Ni-10 at. $\% \mathrm{Cr}$ sample as a function of applied field. The inset shows $T_{S}$ as a function of applied field for all samples. (c) Variation of coercivity $\left(H_{c}\right)$ as a function of temperature for all $\mathrm{Ni}_{1-x} \mathrm{Cr}_{x}(0 \leqslant x \leqslant 0.15)$ nanoparticles.

ordering, where the anti-FM (due to the $\mathrm{Cr}$ segregates) and FM (due to Ni-rich particle cores) clusters are competing. Therefore, $T_{S}$ can be either the cluster-glass transition $\left(T_{G}\right)$ temperature or the blocking temperature $\left(T_{B}\right)$. 
To discern between the cluster-glass or blocking property, the thermal dependence of ZFC/FC curves was obtained under several applied magnetic fields ( $\left.H_{\text {app }}=50-10 \mathrm{kOe}\right)$, as shown in Fig. 7(b) for one representative sample of $\mathrm{Ni}-10 \% \mathrm{Cr}$. As $H_{\text {app }}$ increases, it substantially changes the shape of the ZFC curves, shifting the $T_{S}$ toward lower values; eventually, the ZFC and FC curves merge at very high $H_{\text {app }}$ values. If the progressive spin blocking of the nanoparticles was governed by the superparamagnetic phenomenon, $T_{S}$ would correspond to $T_{B}$, and its field dependence would be given by $T_{B} \propto V\left(H_{K}-H\right)^{2}$ [53], where $V$ is the volume of the spin cluster, $H_{K}$ is the anisotropic field, and $H$ is the applied field. However, the magnetic state of our nanoparticles can be better approximated by fitting the field-dependent $T_{S}$ with the expression [54]

$$
T_{S}(H)=T_{\max }(0)\left[1-\left(\frac{H}{H_{G}}\right)^{n}\right],
$$

where $T_{\max }(0)$ represents the freezing temperature (which is $T_{G}$ in a vanishing magnetic field, $H=0$ ), and $H_{G}$ is a "glassy" parameter, pointing to magnetic fields effectively suppressing the glassy magnetic state of the compound (around 1-2 kOe). From the fitting shown in the inset of Fig. 7(b), the obtained values of parameter $n$ range between 0.07 and 0.37 , revealing cluster-glass rather than canonical spin-glass behavior: $n<2 / 3$ is the fingerprint of cluster-glass formation, whereas for the so-called de Almeida-Thouless line, characteristic of spin glasses, $n=2 / 3$ [52,55]. Figure 7(c) shows a comparison of $H_{C}$ values for nanoparticles of all $\mathrm{Cr}$ concentrations as a function of temperature. The curves were fitted with very good agreement to an exponential law [i.e., $H_{C}(T)=H_{C}(0) \exp (-\alpha T)+C$, with the exponent fitting parameter, $\alpha$, in the range $\left.0.03-15 \mathrm{~K}^{-1}\right]$, rather than to the slower decay approach of interacting superparamagnetic nanoclusters [i.e., $H_{C}(T)=H_{C}(0)\left(a-b T^{\frac{1}{3}}\right)$ ] [56]. A similar exponential dependence of $H_{C}(T)$ was reported in Ni nanoparticles and rare-earth transition-metal magnets, where strong FM exchange and random magnetic anisotropy are present [57].

Overall, the obtained data support the hypothesis of a cluster-glass ground state of the $\mathrm{Ni}_{1-x} \mathrm{Cr}_{x}(0 \leqslant x \leqslant 0.15)$ nanoparticles rather than that of a conventional spin-glass state: (i) the irreversibility temperature $T_{i r r}$ and the glass-freezing temperature $T_{S}$ do not coincide in the applied low magnetic fields; (ii) the approximations of $T_{S}(H)$ in low magnetic fields $H<3 \mathrm{kOe}$ give the parameter value $n=0.37 \pm 0.1$, which agrees with cluster-glass behavior; (iii) $H_{C}(T)$ follows a rapid exponential behavior below $T_{S}(H)$; and (iv) FC curves exhibit no plateaus below $T_{G}$.

\section{F. Construction of the nanophase diagram}

Changes in the interrelated magnetic and structural properties of nanoalloys with respect to those of the parental bulk alloy can be readily explained with the help of corresponding nanophase diagrams. However, due to size-related inhomogeneity specific to each studied system, these are difficult to construct and, hence, scarce. Here, to highlight the complexity of alloying at the nanoscale, we constructed a nanophase diagram at the magnetic end of the compositional range of

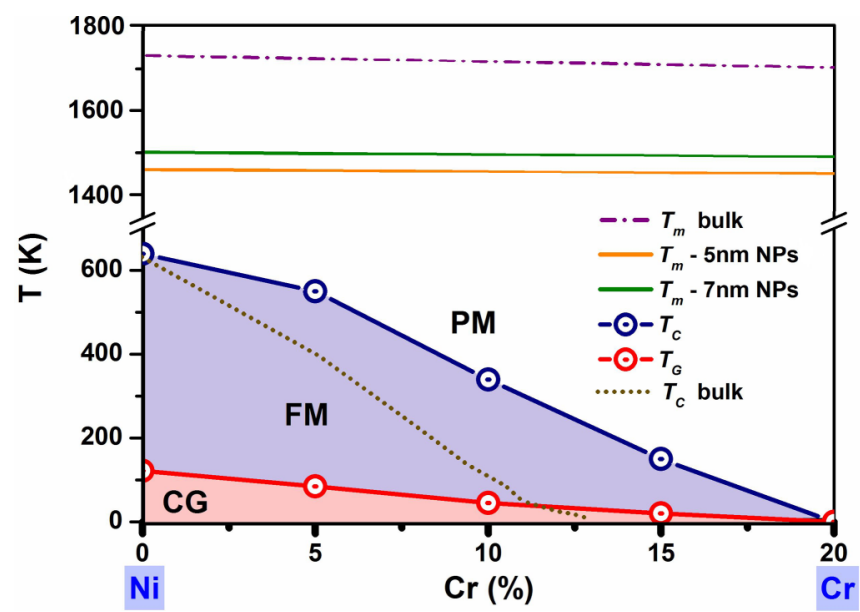

FIG. 8. Magnetic phase diagram for the $\mathrm{Ni}_{1-x} \mathrm{Cr}_{x}$ nanoclusters as a function of $\mathrm{Cr}$ atomic concentration. Size-dependent melting point depression was calculated by MD via corresponding caloric curves. The Curie $\left(T_{C}\right)$ and cluster-glass $\left(T_{G}\right)$ temperatures are indicated by blue and red open circles, respectively. $T_{G}$ is estimated from expression (2) at $H=0$. The blue region represents the long-rangeFM order below $T_{C}$, covering a larger area compared to the bulk data (shown by a black dotted line) as a consequence of $\mathrm{Cr}$ segregation. The pink region below $T_{G}$, which led the system from the FM state to the cluster-glass state, is detected at very low temperatures.

$\mathrm{NiCr}$ nanoparticles, i.e., $0-20$ at. \% Cr, presented in Fig. 8, based on our MD (melting points) and experimental results (magnetic state transitions). More specifically, MD caloric curves, temperature dependence of magnetization (at cooling rate $2 \mathrm{~K} / \mathrm{min}$ ), and $\mathrm{x}$-ray and electron-diffraction studies were used for the proposed magnetic ground-state phase diagram.

The Curie temperature $T_{C}$ decreases continuously (blue solid line) with increasing $\mathrm{Cr}$ content at a slower rate compared to the bulk $\mathrm{Ni}_{1-x} \mathrm{Cr}_{x}$ (black dotted line), due to the presence of $\mathrm{Cr}$ segregates (note that the $T_{C}$ for $\mathrm{Ni}_{0.8} \mathrm{Cr}_{0.2}$ samples was extrapolated from lower $\mathrm{Cr}$ concentrations). As hinted above, the slope of the FM-to-PM transformation line changes at a concentration of 5 at. $\% \mathrm{Cr}$, indicating the transition from full to partial $\mathrm{Cr}$ segregation. A transition can be observed from the FM state of $\mathrm{Ni}$ to the PM state for $x \approx 0.20$ in $\mathrm{Ni}_{1-x} \mathrm{Cr}_{x}$ nanoalloys, due to modifications in the conduction band produced by the substitution of $\mathrm{Ni}$ by $\mathrm{Cr}$ atoms. Accordingly, the $\mathrm{FM}$ order is predicted to vanish close to $x \approx 0.20$, whereas for bulk $\mathrm{Ni}_{1-x} \mathrm{Cr}_{x}$ this happens earlier, at $x \approx 0.13$. This roughly confirms that due to $\mathrm{Cr}$ segregation, one needs an additional $\sim 5 \% \mathrm{Cr}$ to obtain bulk $T_{C}$ values when using the present deposition method.

Furthermore, a cluster-glass-like state (CG, below the red solid line) was found at temperatures below the FM ordered state $\left(T_{G}<T_{C}\right)$. In all spin glass systems, the common denominator is some random frustration of the exchange interactions leading to many nearly degenerate ground states. In the present case, it is the surface spin disorder and dipolar interaction of competing FM and anti-FM bonds, combined with the randomness due to the arbitrary locations of the magnetic particles, which generate the cluster-glass state in an ensemble of interacting single-domain particles. 


\section{SUMMARY AND CONCLUSIONS}

Control of the Curie temperature $T_{C}$ at the nanoscale is imperative for a wide variety of potential magnetic applications; however, the difficulty of controlling simultaneously the structural homogeneity and magnetic ordering of nanoalloys has posed a formidable challenge. Herein, we studied the effect of $\mathrm{Cr}$ doping on the structural and corresponding magnetic properties of $\mathrm{Ni}_{1-x} \mathrm{Cr}_{x}(0 \leqslant x \leqslant 0.15)$ nanoparticles grown by cluster beam deposition, a versatile method that allows onestep, chemical-free fabrication of size-selected nanoparticles of stoichiometric compositions. We demonstrated the possibility to readily tune the $T_{C}$ within a wide temperature range via modifying a single parameter, i.e., the $\mathrm{Cr}$ concentration of the nanoparticles. Combined experimental and computational analysis of the growth mechanism and the structure of the nanoparticles showed preferential full surface segregation of $\mathrm{Cr}$ up to 5 at. $\% \mathrm{Cr}$, but, unexpectedly, the presence of residual $\mathrm{Cr}$ inside the Ni-rich cores for higher concentrations. We explained the presence of residual $\mathrm{Cr}$ in the nanoparticle cores on the grounds of modifications in the local environment of individual $\mathrm{Cr}$ atoms. This phenomenon, also reflected in our constructed magnetic nanophase diagram, dictates the various competitive interactions present inside a system consisting of magnetic nanoparticles, determining its cluster glass magnetic state at low temperatures. Further elucidation of the $\mathrm{Cr}$ segregation effect on magnetic properties may open pathways toward manipulating the coupling between FM and anti-FM ordering of core/shell nanoclusters for exchange-bias-based devices in order to overcome their superparamagnetic limit.

\section{ACKNOWLEDGMENTS}

The authors would like to thank R. E. Diaz for her help with TEM measurements, C. Lal for his help with XPS measurements, and J. Vernieres for useful discussion and advice during the preparation of the manuscript. Work performed at OIST was supported by funding from the Okinawa Institute of Science and Technology Graduate University. We are also grateful to the Finnish IT Centre for Science CSC and the Finnish Grid Infrastructure (FGI) for grants of computation time.
[1] M. Dobrowolska, K. Tivakornsasithorn, X. Liu, J. K. Furdyna, M. Berciu, K. M. Yu, and W. Walukiewicz, Nat. Mater. 11, 444 (2012).

[2] M. J. Besnus, Y. Gottehrer, and G. Munschy, Phys. Status Solidi B 49, 597 (1972).

[3] J. Zhao, E. Baibuz, J. Vernieres, P. Grammatikopoulos, V. Jansson, M. Nagel, S. Steinhauer, M. Sowwan, A. Kuronen, K. Nordlund, and F. Djurabekova, ACS Nano 10, 4684 (2016).

[4] Y. Akin, I. M. Obaidat, B. Issa, and Y. Haik, Cryst. Res. Technol. 44, 386 (2009).

[5] M. Kaur, Q. Dai, M. Bowden, M. H. Engelhard, Y. Wu, J. Tang, and Y. Qiang, Nanoscale 5, 7872 (2013).

[6] L. Pathade, T. L. Doane, R. D. Slaton, and M. M. Maye, J. Phys. Chem. C 120, 22035 (2016).

[7] J. A. Sundararajan, T. Schimel, P. Kaur, Y. Qiang, C. Wang, D. R. Baer, and S. M. Bruemmer, 11th IEEE Conference on Nanotechnology (IEEE-NANO), Portland, OR (IEEE, Piscataway, NJ, 2011).

[8] M. Bohra, P. Grammatikopoulos, R. E. Diaz, V. Singh, J. Zhao, J.-F. Bobo, A. Kuronen, F. Djurabekova, K. Nordlund, and M. Sowwan, Chem. Mater. 27, 3216 (2015).

[9] C.-M. Wang, A. Genc, H. Cheng, L. Pullan, D. R. Baer, and S. M. Bruemmer, Sci. Rep. 4, 3683 (2014).

[10] C.-M. Wang, D. R. Baer, S. M. Bruemmer, M. H. Engelhard, M. E. Bowden, J. A. Sundararajan, and Y. J. Qiang, Nanosci. Nanotechnol. 11, 8488 (2011).

[11] I. Ban, J. Stergar, M. Drofenik, G. Ferk, and D. Makovec, Acta Chim. Slov. 60, 750 (2013).

[12] H. Amekura, Y. Fudamoto, Y. Takeda, and N. Kishimoto, Phys. Rev. B 71, 172404 (2005).

[13] M. Bohra, V. Singh, P. Grammatikopoulos, E. Toulkeridou, R. E. Diaz, J.-F. Bobo, and M. Sowwan, Sci. Rep. 6, 19153 (2016).

[14] S. F. Fischer, S. N. Kaul, and H. J. Kronmüller, Magn. Magn. Mater. 226-230, 540 (2001).

[15] M. Ruano, M. Díaz, L. Martınez, E. Navarro, E. Román, M. García-Hernandez, A. Espinosa, C. Ballesteros, R. Fermento, and Y. Huttel, Phys. Chem. Chem. Phys. 15, 316 (2013).
[16] C. Cherian Lukose, G. Zoppi, and M. Birkett, IOP Conf. Ser.: Mater. Sci. Eng. 104, 012003 (2015).

[17] P. Grammatikopoulos, S. Steinhauer, J. Vernieres, V. Singh, and M. Sowwan, Adv. Phys.: X 1, 81 (2016).

[18] M. Bohra, V. Singh, M. Sowwan, J.-F. Bobo, C.-J. Chung, and B. Clemens, J. Phys. D 47, 305002 (2014).

[19] G. Bonny, N. Castin, and D. Terentyev, Model. Simul. Mater. Sci. Eng. 21, 085004 (2013).

[20] J. F. Ziegler, J. P. Biersack, and U. Littmark, The Stopping and Range of Ions in Matter (Pergamon, New York, 1985).

[21] C. Kittel, Introduction to Solid State Physics, 6th ed. (Wiley, New York, 1986).

[22] K. Nordlund, PARCAS computer code, Purdue University, West Lafayette, IN, 2006.

[23] K. Nordlund, M. Ghaly, R. S. Averback, M. Caturla, T. Diaz de la Rubia, and J. Tarus, Phys. Rev. B 57, 7556 (1998).

[24] M. Ghaly, K. Nordlund, and R. S. Averback, Philos. Mag. A 79, 795 (1999).

[25] H. J. C. Berendsen, J. P. M. Postma, W. F. van Gunsteren, A. DiNola, and J. R. Haak, J. Chem. Phys. 81, 3684 (1984).

[26] S. J. Plimpton, J. Comput. Phys. 117, 1 (1995).

[27] S. Nosé, J. Chem. Phys. 81, 511 (1984).

[28] W. G. Hoover, Phys. Rev. A 31, 1695 (1985).

[29] G. Henkelman and H. Jónsson, J. Chem. Phys. 113, 9978 (2000).

[30] G. Henkelman, B. P. Uberuaga, and H. Jónsson, J. Chem. Phys. 113, 9901 (2000).

[31] D. Sheppard, R. Terrell, and G. Henkelman, J. Chem. Phys. 128 134106 (2008).

[32] Classical and Quantum Dynamics in Condensed Phase Simulations, edited by B. J. Berne, G. Ciccotti, and D. F. Coker (World Scientific, Singapore, 1998).

[33] P. Grammatikopoulos, J. Kioseoglou, A. Galea, J. Vernieres, M. Benelmekki, R. E. Diaz, and M. Sowwan, Nanoscale 8, 9780 (2016).

[34] P. Grammatikopoulos and M. Sowwan, in Nanostructured Semiconductors: Amorphization and Thermal Properties, edited by K. Termentzidis (CRC Press, Boca Raton, 2017). 
[35] P. Krashnochtchekov, K. Albe, and R. S. Averback, Z. Metallk. 94, 1098 (2003).

[36] R. T. Pack, R. B. Walker, and B. K. Kendrick, J. Chem. Phys. 109, 6701 (1998).

[37] R. T. Pack, R. B. Walker, and B. K. Kendrick, J. Chem. Phys. 109, 6714 (1998).

[38] A. Stukowski, Modell. Simul. Mater. Sci. Eng. 18, 015012 (2010).

[39] V. Singh, C. Cassidy, P. Grammatikopoulos, F. Djurabekova, K. Nordlund, and M. Sowwan, J. Phys. Chem. C 118, 13869 (2014).

[40] V. Suresh Babu, A. S. Pavlovic, and M. S. Seehra, J. Appl. Phys. 79, 5230 (1996).

[41] T. Ohgai, Y. Tanaka, and T. J. Fujimaru, Appl. Electrochem. 42, 893 (2012).

[42] L. Peng, R. P. Van Duyne, and L. D. Marks, J. Phys. Chem. Lett. 6, 1930 (2015).

[43] M. J. Hÿtch, E. Snoeck, and R. Kilaas, Ultramicroscopy 74, 131 (1998).

[44] J. Kioseoglou, G. P. Dimitrakopulos, Ph. Komninou, Th. Kehagias, and Th. Karakostas, Mixed Phys. Status Solidi A 203, 2156 (2006).

[45] P. Stadelmann, Ultramicroscopy 21, 131 (1987).
[46] See Supplemental Material at http://link.aps.org/supplemental/ 10.1103/PhysRevMaterials.1.066001 for details on the calculation of the overall magnetization of our thin film.

[47] M. Bohra, S. Prasad, N. Venkataramani, S. C. Sahoo, N. Kumar, and R. Krishnan, IEEE Trans. Magn. 49, 4249 (2014).

[48] M. D. Kuz'min, M. Richter, and A. N. Yaresko, Phys. Rev. B 73, 100401 (2006).

[49] J. R. Thompson, A. Goyal, D. K. Christen, and D. M. Kroeger, Physica C 370, 169 (2002).

[50] V. Chaudhary and R. V. Ramanujan, Sci. Rep. 6, 35156 (2016).

[51] M. Bohra and S. C. Sahoo, J. Alloys Compd. 699, 1118 (2017).

[52] T. Elovaara, H. Huhtinen, S. Majumdar, and P. Paturi, J. Phys.: Condens. Matter 26, 266005 (2014).

[53] S. Mukherjee, A. K. Pal, S. Bhattacharya, and J. Raittila, Phys. Rev. B 74, 104413 (2006).

[54] L. He, W. Zheng, W. Zhou, H. Du, C. Chen, and L. Guo, J. Phys.: Condens. Matter 19, 036216 (2007).

[55] E. Fertman, S. Dolya, V. Desnenko, A. Beznosov, M. Kajňaková, and A. Feher, J. Magn. Magn. Mater. 324, 3213 (2012).

[56] S. Vitta, J. Appl. Phys. 101, 063901 (2007).

[57] V. Singh, V. Srinivas, M. Ranot, S. Angappane, and J.-G. Park, Phys. Rev. B 82, 054417 (2010). 